\title{
Optimal Gut and Survival in AGP-Free Broilers: Effects of Medium-Chain Glycerides and Phytogenics
}

\author{
Kirsten De Keyser, DVM, PhD* \\ New Business Development, Belgium \\ *Corresponding author: Kirsten De Keyser, DVM, PhD, Eugeen Meeusstraat 6, 2170 Merksem, Belgium \\ Submission: 㘹 October 13, 2017; Published: 眥 January 30, 2018
}

\section{Opinion}

The key to optimal animal production is a balanced gut. Performance pressure renders broilers vulnerable to infectious and/or non-infectious triggers. With the European withdrawal of antimicrobial growth promoters (AGP) in 2006, enteric broiler diseases became more prevalent in the EU. A common syndrome causing intestinal health aberrance is called 'dysbiosis' or 'dysbacteriosis' [1]. This condition hampers a proper digestion thus compromises productivity, survival and well being whilst increasing time and cost of therapeutic treatment. More recently, also the therapeutic antibiotic use is monitored in many EU countries. For instance in Belgium (AMCRA, Belgium, EU), stringent goals have been set towards 2020 and further on, aiming to reduce and preferable abandon antimicrobials in animal husbandry. All of this to monitor and diminish antibiotic resistance in production animals to safeguard animal and human health and well-being.

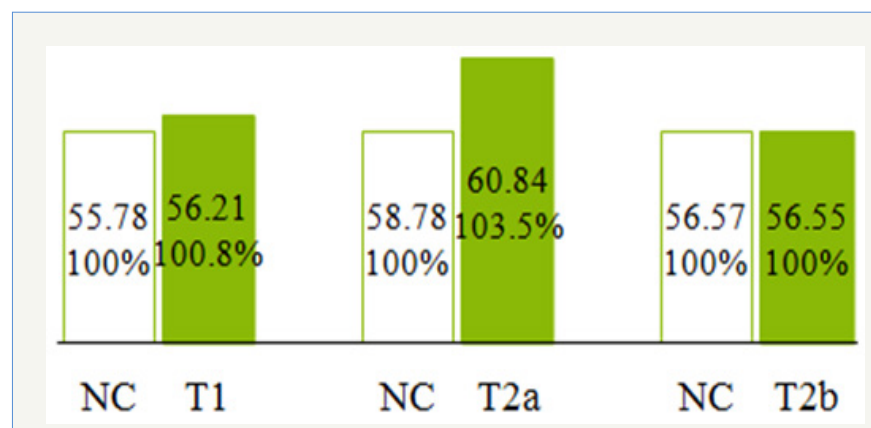

Figure 1: Growth rate (g/day).

Since the late 90's, AVEVE Biochem (Belgium, EU) cooperated with Ghent University (Belgium, EU) to investigate natural gut regulator molecules for in-feed use. The concepts that were created foresaw the EU AGP-ban of 2006 and are in accordance with the 2020 outlook written above. Present study reports on the combined effect of medium-chain glycerides (MCG) and phytogenics. Indeed, in 2003, these MCG were already proposed as an alternative for in feed antibiotics by Decuypere and Dierick (UGhent, Ghent, Belgium, EU) [2]. MCG are esters of glycerol and medium chain fatty acids, which are fatty acids with a carbon chain length of 6 to 12 atoms.
MCG has inherent 'slow release' properties without a repellent smell or taste [3]. This is a concept from nature, cfr. milk lipids that are converted to mono-glycerides and fatty acids by lipase in suckling neonates. Such lipids serve as a defense system against microbial infections at the gut mucosa [4]. Furthermore, at the same UGhent research group, Prof. Michiels J [5] studied the effects of phytogenics as f.i. carvacrol and thymol on gut bacteria in pigs $[5,6]$.
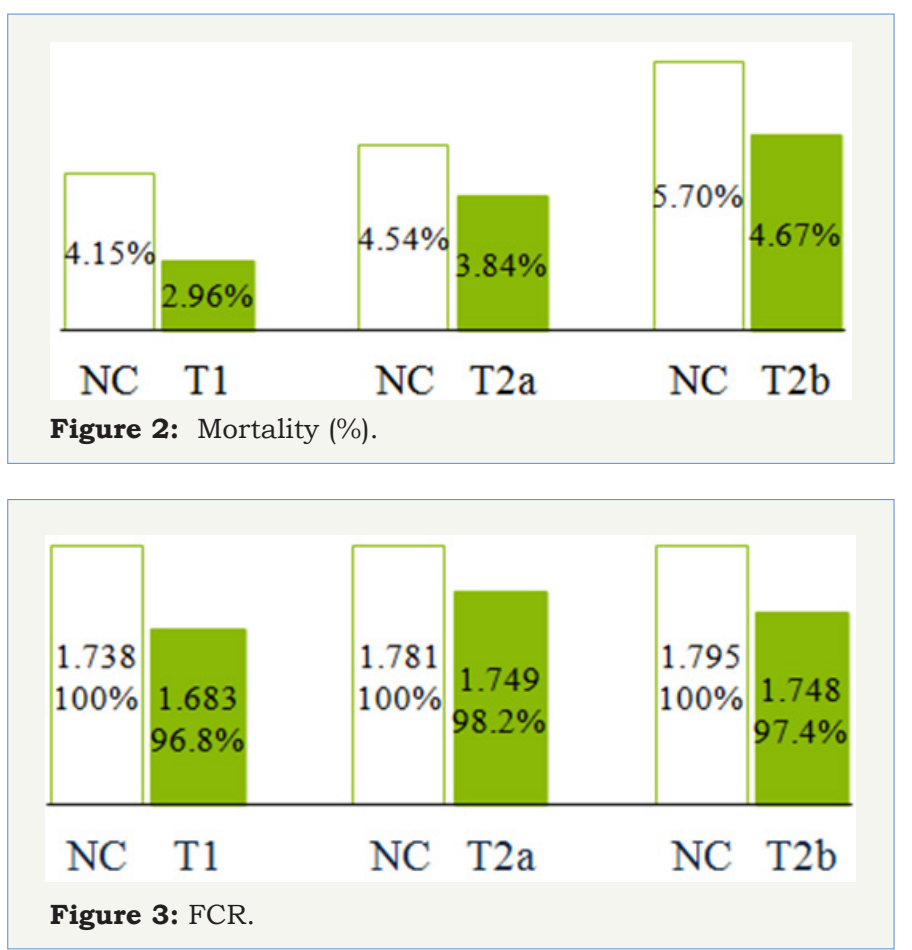

Here, results our of a broiler field case are presented. Birds were fed AGP-free feed. The concept of MCG plus phytogenics at different dosage rates (brand AveMix®, AVEVE Biochem, Merksem, Belgium, EU) was added for optimal gut health control. Growth rate (Figure 1), feed conversion rate and mortality (Figure 2) were assessed in Ross 308 broilers as parameters for optimal broiler health and productivity. One group of 61460 birds received a 
$0.10 \%$ of the concept (T1), two successive groups of 84135 and 84100 birds $0.06 \%$ (T2a and T2b). Each group was compared to a non supplemented control group (NC). We recorded feed intake (g/day), weight gain (g/day), and mortality (\%) (Figure 2) and calculated FCR (Figure 3) and FCR1500 (Figure 4).

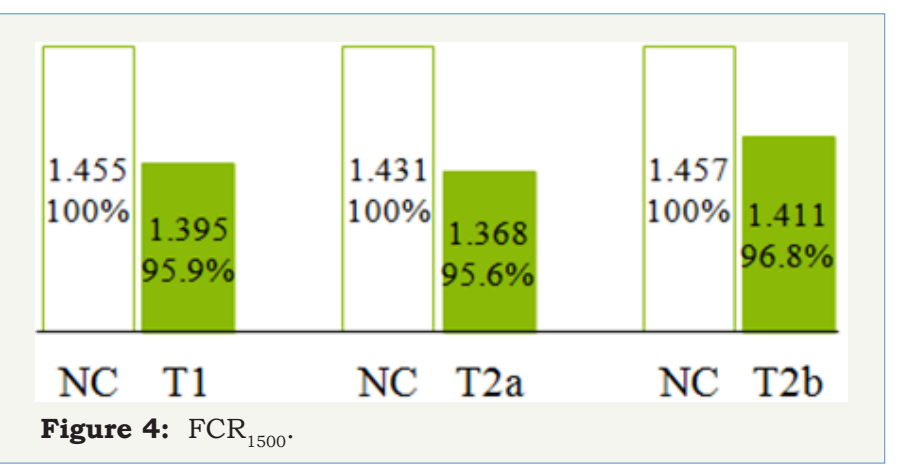

Mortality was 29, 15 and 18\% lower than NC in respectively T1, T2a and T2b. This indicates that birds fed the concept were able to cope much better with intensive production than NC in the absence of AGP. Also FCR and FCR1500 of T1 and two T2 groups were lower than NC, where FCR and FCR1500 were similar for T1 and $\mathrm{T} 2 \mathrm{a}$ and $\mathrm{b}$. These results demonstrate that the most important economic production trait 'feed to gain' has improved compared to NC using MCG plus phytogenics. This study reports on the benefits of presented AveMix ${ }^{\circledR}$ concept for broiler production using $1000 \mathrm{~g}$ or even 600g per ton of AGP free compound feed. This concept from nature is in accordance with regulatory objectives for AGP-free feed and enables a drastic diminishing of antimicrobials in animal production. It thereby supports the 'one health' approach to reduce animal and human antibiotic resistance.

\section{References}

1. Teirlynck E, Gussem MD, Dewulf J, Haesebrouck F, Ducatelle R, et al. (2011) Morphometric evaluation of 'dysbacteriosis' in broilers. Avian Pathol 40(2): 139-144.

2. Decuypere JA, Dierick NA (2003) The combined use of triacylglycerols containing medium-chain fatty acids and exogenous lipolytic enzymes as an alternative to in-feed antibiotics in piglets: concept, possibilities and limitations. An overview. Nutr Res Rev 16(2): 193-209.

3. Messens W, Goris J, Dierick N, Herman L, Heyndrickx M (2010) Inhibition of Salmonella typhimurium by medium-chain fatty acids in an in vitro simulation of the porcine cecum. Vet Microbiol 141(1-2): 73-80.

4. Isaacs CE (2001) The antimicrobial function of milk lipids. Adv Nutr Res 10: 271-285.

5. Michiels J (2009) Effect of essential oils on gut bacteria and functionality in the pig. Dissertation, Faculty of Bioscience Engineering, Ghent University, Ghent, Belgium.

6. Michiels J, Missotten J, Van Hoorick A, Ovyn A, Fremaut D, et al. (2010) Effects of dose and formulation of carvacrol and thymol on bacteria and some functional traits of the gut in piglets after weaning. Arch Anim Nutr 64(2): 136-154. 